\title{
ACESSO LIVRE À INFORMAÇÃO CIENTÍFICA EM PAÍSES EM DESENVOLVIMENTO: O CASO DOS REPOSITÓRIOS "SABER" (MOÇAMBIQUE) E DIGITAL DA UFMG (BRASIL)
}

\author{
Ranito Zambo Waete \\ Maria Aparecida Mourai \\ Manuel Valente Mangue iii
}

\begin{abstract}
Resumo: Os repositórios institucionais digitais de acesso livre têm proporcionado um forte debate na literatura especializada em publicações eletrônicas; mais focados nas questões sobre o movimento de acesso livre à informação científica. É tema, portanto, da preocupação de todos os atores da comunidade científica, nomeadamente docentes e pesquisadores, editores científicos, comunidades científicas, bibliotecas universitárias e provedores de acesso e de serviços. Descreve-se os repositórios institucionais de acesso livre à informação científica "Saber" (Moçambique) e Digital da UFMG (Brasil) que têm como principal objetivo preservar, organizar, divulgar e disseminar a produção científica das instituições de ensino superior e de pesquisa, utilizando o software livre DSpace.
\end{abstract}

Palavras-chave: Repositórios Digitais. Repositórios Institucionais. Acesso Livre.

\section{OPEN ACCESS TO SCIENTIFIC INFORMATION IN DEVELOPING COUNTRIES: THE CASE OF REPOSITORY "SABER" (MOZAMBIQUE) AND DIGITAL UFMG (BRAZIL)}

\begin{abstract}
The institutional digital repositories of open access have supported a fruitful debate in the literature about electronic publications with focus on the movement of open access to scientific information. The institutional digital repositories are concern to all members of the scientific community, including professors, and researchers, scientific publishers, scientific communities, academic libraries and access providers and services. It describes the institutional repositories of open access to scientific information "Saber" (Mozambique) and Digital UFMG (Brazil) whose main objectives are to preserve, organize, publicize and disseminate the scientific production of institutions of higher education and research, using DSpace open source software.
\end{abstract}

Keywords: Digital Repositories. Institutional Repositories. Open Access.

\footnotetext{
${ }^{\mathrm{i}}$ Universidade Federal de Minas Gerais - rzwaete@gmail.com

ii Universidade Federal de Minas Gerais - mamoura@eci.ufmg.br

iii Universidade Eduardo Mondlane (Moçambique). - $\underline{\text { mvmangue@yahoo.com.br }}$
} 


\section{INTRODUÇÃO}

A informação científica é o insumo básico para o desenvolvimento científico e tecnológico em todos os países. No entanto, a comunidade científica enfrenta dificuldades no acesso à informação, se considerado o modelo tradicional de publicação e circulação científica. Tradicionalmente, os artigos ou trabalhos científicos são publicados em revistas especializadas, e a forma de acesso a esses trabalhos dá-se mediante assinatura das publicações pelas bibliotecas ou pelo pesquisador. ${ }^{3}$

Mangue (2009) salienta que, "as barreiras, sobretudo financeiras, para o acesso a essas publicações têm sido cada vez maiores, contrariando inclusive as expectativas avançadas por alguns autores que acreditavam na redução dos custos de publicação e de acesso à informação científica, graças à Internet". Nesse sentido, o acesso livre pode facilitar a circulação e o acesso a informação científica e promover o surgimento de novas alternativas para a comunicação científica. Os repositórios institucionais são exemplo disso.

Porém, a submissão de trabalhos acadêmicos em repositórios de acesso livre (RAL) tem sido alvo de críticas no meio acadêmico, relacionadas, sobretudo, aos direitos e ao papel da publicação periódica científica como veículo privilegiado para a comunicação científica.

Embora a temática referente à comunicação científica seja central no contexto estudado, no âmbito desse trabalho serão analisadas as contribuições de duas experiências oriundas do Brasil e de Moçambique relativas à promoção do acesso livre (open access) à informação científica através de repositórios digitais.

O presente artigo representa um esforço de articulação bilateral de colocar em debate o acesso livre à informação científica no Brasil e em Moçambique, via ou através dos repositórios institucionais "Saber" e digital da UFMG.

\section{O PAPEL DA INICIATIVA OPEN ACCESS NA PROMOÇÃO DA INFORMAÇÃO CIENTÍFICA EM PAÍSES EM DESENVOLVIMENTO}

Permitir a livre utilização dos resultados da pesquisa científica contida em revistas especializadas é a principal meta do Acesso Livre (AL). Embora o interesse neste sentido seja

\footnotetext{
3 MANIFESTO brasileiro de apoio ao acesso livre à informação científica. Disponível em: http://kuramoto.files.wordpress.com/2008/09/manifesto-sobre-o-acesso-livre-a-informacao-cientifica.pdf. Acessado em: 13 maio 2010. 196 
extensivo a outros tipos de materiais (relatórios técnicos, dissertações, teses, etc.), as atenções na abordagem deste conceito estão quase todas voltadas para as revistas científicas devido à sua centralidade no processo de difusão e circulação do conhecimento.

No que diz respeito aos conceitos-chave, é importante salientar que o AL não é sinônimo de enfraquecimento da qualidade das revistas. Embora livre, de modo algum o AL dispensa os mecanismos ou Sistema de Avaliação pelos Pares (peer-review). Na realidade, o AL mantém, na sua essência, todas as exigências inerentes à publicação tradicional de revistas científicas, excetuando-se na forma de acesso que, segundo autores, deve ser aberta e sem restrições; forma essa propiciada em grande medida pela Internet. (SANZ-VALERO; VEIGA DE CABO, 2007; CANESSA; ZENNARO, 2008).

O sistema de publicação tradicional e o AL, portanto, são sinônimos, conquanto que a primeira preserve as suas principais características e o AL circule em revistas de acesso aberto. Ainda no que se refere às questões relacionadas com a qualidade das revistas, vale destacar que o AL é compatível com o copyright e com os demais critérios adotados nas formas tradicionais de circulação da produção científica, dentre eles a preocupação com a normalização, a indexação em bases de dados internacionais.

\subsection{Acesso livre: modalidades}

Como se fez referência, não só as revistas constituem objeto do AL, outros materiais são comuns em RI - tais como dissertações, teses ou outros documentos que resultam de pesquisas científicas. Para alguns autores, o AL torna-se cada vez mais abrangente, seja em termos de tipos de documentos disponibilizados, as comunidades científicas envolvidas e as modalidades de publicação. Para Bianco et al (2007), é cada vez maior o número de comunidades científicas que se "reúne" em torno desta filosofia para a divulgação de suas pesquisas, além das instituições de fomento que recomendam, como política, que os resultados das pesquisas financiadas devam ser depositados em plataformas de acesso livre. (SANZ-VALERO; VEIGA DE CABO, 2007)

No que tange às modalidades, Canessa; Zennaro (2008) apresentam pelo menos dois principais tipos de publicação no AL, a saber: (1) o Gold Open Access, no qual o autor publica em uma revista cujo acesso é irrestrições; e (2) o Green Open Access, no qual o artigo é publicado em uma revista de acesso restrito e posteriormente disponibilizado num repositório de acesso aberto. Associado a essa modalidade, Mueller (2006, p. 32) descreve uma variante, na qual o "autor (ou sua instituição) paga ao editor para que seu artigo possa ser 
acessado livremente." Outra forma variante do Green Open Access - na qual o autor deposita pessoalmente o seu trabalho - recebe a designação de auto-arquivamento e tem o objetivo de acelerar e aumentar o impacto da pesquisa científica (CANESSA; ZENNARO, 2008, p. 6).

O depósito ou circulação de determinado documento antes mesmo de este ter sido avaliado pelos pares recebe a designação de preprint, cujo maior propósito é o de acelerar e promover o debate entre cientistas. Tão logo a avaliação aconteça, o artigo passa à postprint.

Essas modalidades não são exclusivas das revistas científicas propriamente ditas, mas abrangem também os repositórios institucionais, que, por definição:

[...] reúnem documentos produzidos na instituição. Por exemplo, repositórios de uma universidade reuniriam toda a produção científica ou acadêmica produzida na universidade, em forma digital, formando coleções de documentos digitais. [...] os repositórios são abertos a todos os interessados, oferecendo meios de busca, identificação e recuperação. Todo o tipo de documento produzido na universidade seria depositado no repositório universitário, como trabalho dos professores e pesquisadores apresentados em congressos e reuniões profissionais, versões de artigos impressos, relatórios de pesquisa, programas de disciplinas e textos elaborados para aulas, trabalhos elaborados por alunos, teses, dissertações, trabalhos de disciplinas e outros. (MUELLER, 2006, p. 32).

\subsection{Tecnologias para o desenvolvimento de plataformas para o $\mathrm{AL}$}

O advento da Internet e suas ferramentas correlatas é, sem dúvida, o principal impulsionador do AL ao permitir aglutinar, num mesmo espaço virtual, pelo menos duas características relacionadas com a disponibilização e acesso à informação: a instantaneidade e a simultaneidade, em que independentemente da localização geográfica, um mesmo trabalho científico pode ter múltiplos acessos, tão logo este seja depositado em rede.

Do ponto de vista tecnológico, o AL está intimamente associado ao conceito de open source, isto é, conjunto de ferramentas de código fonte também aberto, passível de manipulação livre e adequação ao contexto de uso. Nessa perspectiva, vários são os aplicativos que podem ser utilizados para o desenvolvimento de plataformas de AL, entre eles o Eprints e o SEER - utilizados essencialmente para a gestão de periódicos - e ferramentas como o Dspace, o Greenstone e o Invenio - utilizadas fundamentalmente para a gestão de repositórios. Privilegia-se o uso de aplicativos de código livre - o MySQL, por exemplo - para a gestão das bases de dados, e às ferramentas como o Apache ou Tomcat, como servidores $W e b$. Sendo várias as opções, a utilização de uma ou outra tecnologia vai depender da "política e conteúdos de cada instituição e dos procedimentos técnicos e administrativos requeridos para implementar essas políticas.” (CANESSA; ZENNARO, 2008, p. 126) 


\subsection{O Acesso livre nos países em desenvolvimento}

Em uma rápida análise do contexto de comunicação da ciência nos países centrais e nos países em desenvolvimento verificam-se claramente as evidências de discrepâncias. A este respeito, dados apresentados por Ondari-Okemwa (2007) mostram que a África do Sul, entre os mais bem posicionados países no continente africano, com 51.738 publicações, publicou, em 10 anos, próximo da metade do que países como o Estados Unidos da América e a Inglaterra publicaram em apenas um ano. A contribuição de um conjunto de países em desenvolvimento, que inclui o Brasil, representa cerca de 10 a 15\% em 10 anos (1997 a 2007), comparativamente a estes dois países centrais.

Contudo, estudos recentes têm mostrado que apesar de ainda permanecer o gap entre os dois blocos, a disponibilização da informação técnico-científica nos países em desenvolvimento tem aumentado, especialmente em acesso aberto. Neste prisma, duas abordagens podem ser encontradas, a saber: (a) algumas revistas antes impressas e de acesso restrito hoje estão disponíveis em acesso aberto; e (b) o surgimento de novos periódicos e repositórios eletrônicos. Rodrigues; Thuns (2010, s/p) reconhecem esse aumento, salientando que "os grandes destaques são o Brasil e a Espanha, países considerados periféricos na publicação científica internacional e que lideram a América e a Europa na quantidade de títulos".

Grande parte desta evolução é atribuída às universidades, como também constata Bolaño; Kobashi; Santos (2006) e na mesma linha Witter (1997), na medida em que as revistas acadêmicas "alimentadas pelo trabalho de pesquisa dos intelectuais vinculados às instituições, privadas e, sobretudo, públicas, representantes de uma esfera produtiva" (p. 126). Esta posição é corroborada pelo trabalho de Rodrigues; Thuns (2010, s/p), segundo o qual, “dos 539 periódicos considerados na amostra, 367 (69\%) são universidades”.

Assim, com a expansão do ensino superior, de um modo geral, e o aumento do número de matrículas na pós-graduação vislumbra-se um crescimento da participação dos países em desenvolvimento no cenário da comunicação científica. A este respeito, Sena (2000), relativamente ao Brasil, mostra um crescimento de perto de 11,7\% já em 1999 no número de matrículas na pós-graduação, com tendência a aumento. Em Moçambique, por exemplo, analisando-se a lista de cursos das principais universidades, verifica-se que até o ano de 2002, os cursos de pós-graduação eram praticamente inexistentes no país, e em 2011, a Universidade Eduardo Mondlane, a maior e mais antiga instituição de ensino superior no país, contava com 31 cursos de mestrado e dois de doutorado. 


\title{
3 REPOSITÓRIOS INSTITUCIONAIS (RI'S)
}

Para Tomaél; Silva (2007), citando Bekaert; Van de Sompel (2006) ${ }^{4}$, os repositórios são:

conjuntos de documentos coletados, organizados e disponibilizados eletronicamente. No contexto específico dos repositórios, os documentos adquirem novas configurações e são denominados objetos digitais ou estrutura de dados digitalmente codificados, composta pelo conteúdo de informação, metadados e identificador. (TOMAÉL; SILVA, 2007, p. 3).

Pode-se categorizar os repositórios em temáticos (focados em determinado domínio do conhecimento) e institucionais (focados na produção de uma instituição específica). Para este trabalho, vamos utilizar a segunda categoria. Portanto, repositórios institucionais (institutional repositories) são coleções que capturam e preservam a produção intelectual de uma ou mais instituições de ensino e de pesquisa (IEP) ou comunidades (CROW, p. 2002) ou ainda um conjunto de serviços que uma IEP oferece aos membros da sua comunidade, a fim de gerenciar e disseminar materiais digitais criados pela instituição e membros da comunidade (LYNCH, p. 2003). Portanto, o que caracteriza os repositórios institucionais é o fato de serem orientados para a informação produzida no ambiente das instituições, sendo desenvolvidos, implementados e mantidos por elas. (TOMAÉL; SILVA, p. 2007)

Neste contexto, Crow (2002); Lynch (2003); Rodrigues [et. al.] (2006) acreditam que “os RI possibilitam reunir, preservar, dar acesso e disseminar boa parte do conhecimento da instituição, contribuindo para aumentar a visibilidade da sua produção científica". Paralelamente a este ponto de vista, Santos; Teixeira; Pinto (2005, p. 2) afirmam que:

\begin{abstract}
nos últimos anos, tem aumentado o número de RI, principalmente dentro da comunidade universitária, com objetivos orientados à gestão, ao armazenamento, à preservação e à divulgação do trabalho intelectual produzido por essa comunidade. É de responsabilidade e interesse das IEP que seus membros disponibilizem os seus trabalhos nesses repositórios institucionais.
\end{abstract}

Para Leite; Costa (2005, p. 8), os RI possuem uma diversidade de tipologia de conteúdos e formatos que podem ser armazenados nos RI, tais como: artigos científicos, livros eletrônicos, capítulos de livros, relatórios técnicos, textos para discussão, teses, dissertações, trabalhos apresentados em conferências, palestra, material de ensino (slides, transparências, texto resumo, resenhas, trabalhos apresentados, entre outros), arquivos multimídia etc. Nesse contexto, o desenvolvimento de RI tem se dado, amplamente, no

\footnotetext{
${ }^{4}$ BEKAERT, J.; VAN DE SOMPEL, H. Augmenting interoperability across scholarly repositories. Report. 2006. Disponível em: http://msc.mellon.org/Meetings/Interop/FinalReport. Acesso em: 22 fev. 2007. 200 
contexto de IEP, a despeito de outras iniciativas em instituições governamentais, principalmente, mas em escala significativamente menor. Os RI nas IEP representam uma nova estratégia que as permite "influenciar de maneira séria e sistemática nas mudanças aceleradas que vêm ocorrendo na produção do saber e na comunicação científica" (LYNCH, 2003). Nesse sentido, visam, em última instância, ao melhoramento do processo de comunicação científica. Para isso, provêm os mecanismos que aumentam tanto a eficácia da preservação da produção intelectual de pesquisadores e instituições acadêmicas quanto à visibilidade de ambos.

Constituem, conforme salienta Crow (2002), "coleções digitais que capturam e preservam a produção intelectual da comunidade de uma única IEP ou de uma comunidade multinstitucional". Desta forma, a necessidade de se preservar digitalmente documentos, que antes se encontravam apenas em papel, e permitir que os mesmos estejam acessíveis a qualquer pessoa, tem levado determinadas instituições, como universidades, bibliotecas e institutos de pesquisa, entre outras, a criar os seus próprios repositórios. (SANTOS; TEIXEIRA; PINTO, 2005)

$\mathrm{Na}$ contemporaneidade, principalmente em países em desenvolvimento, é notório que a grande parte da informação científica produzida nas IESI não está disponível para a comunidade acadêmica e científica. Isto ocorre em função de vários aspectos que perpassam o complexo ambiente da comunicação científica, dentre eles, a morosidade dos processos de publicação, os custos envolvidos na publicação de documentos, a grande quantidade de documentos impressos e eletrônicos dispersos nas coleções. A implementação de repositórios digitais ou repositórios de acesso livre a informação científica é uma das formas que estas instituições dispõem para minimizar a falta de visibilidade de sua produção intelectual. (TOMAÉL; SILVA, 2007)

\subsection{Repositório digital da UFMG}

Na última década a produção e a comunicação científica tornaram-se mais dinâmicas e complexas em virtude da intensificação do uso das tecnologias da informação em rede e da implementação de dispositivos de comunicação direta entre os pesquisadores e seus pares e a comunidade em geral. Dentre os dispositivos utilizados atualmente pelos pesquisadores destacam-se os blogs científicos, as plataformas digitais de compartilhamento de documentos científicos, as comunidades virtuais de prática, os fóruns de discussão, os periódicos eletrônicos e os repositórios digitais. 
Apesar da diversidade de mecanismos de produção, tratamento e difusão da informação científica, lamentavelmente, ainda é grande o desperdício de recursos informacionais presentes nos documentos gerados ao longo da pesquisa que são utilizados com exclusividade e depois descartados.

Segundo dados do RCAAP "apenas cerca de 10\% da produção científica institucional mundial é auto-arquivada espontaneamente hoje em dia em repositórios e páginas Web", o que representa a perda de uma grande capacidade científica e cultural. Nesse contexto, o compartilhamento de acervos em rede pode viabilizar uma economia significativa, visto que o reuso dos dados e a estruturação de bases de informação comuns enseja o conhecimento mais amplo das pesquisas desenvolvidas na instituição e o desenvolvimento de uma cultura colaborativa em termos do acesso à informação.

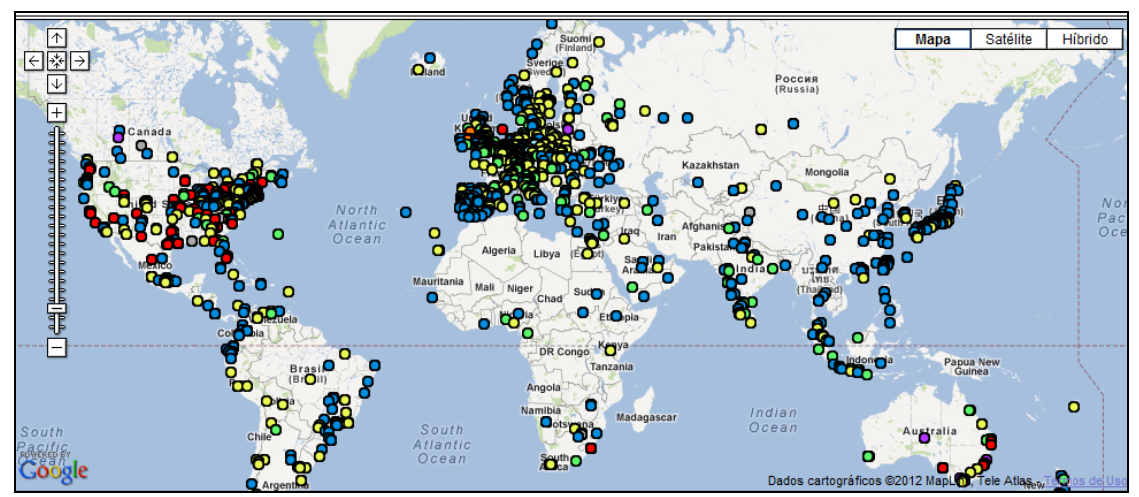

Figura 1: Distribuição e tipos de repositórios no mundo

Fonte: Repository66.org ${ }^{5}$

No Brasil o debate sobre repositórios institucionais de pesquisa teve início de 2007 com a apresentação do projeto de lei proposto por Rodrigo Rollemberg que tinha como propósito regular os processos de disseminação da pesquisa científica nacional. O projeto se baseou na seguinte justificativa:

a universidade hoje dispõe de meios acessíveis, criativos e baratos para superar os muros invisíveis que a separam de parte da sociedade. Iniciativas como o estímulo ao acesso aberto, a criação de um repositório de conteúdos digitais e a elaboração de uma política consistente com respeito à divulgação de conteúdos digitais vêm ao encontro da demanda dos docentes de difundir sua produção intelectual e de ter acesso facilitado às pesquisas dos colegas. Além disso, a promoção de mecanismos de acesso aberto dá mais visibilidade e transparência àquilo que é produzido pela universidade, reforçando sua função de servir à sociedade ao promover o conhecimento científico e a difusão cultural. ${ }^{6}$

\footnotetext{
${ }^{5} \mathrm{http}: / /$ maps.repository66.org/

${ }^{6} \mathrm{http}: / /$ www.camara.gov.br/sileg/integras/461698.pdf 202 
Diante desse desafio e das inúmeras iniciativas acadêmicas articuladas em nível mundial, a UFMG decidiu dar início à integração de seus acervos digitais de acesso aberto através da criação de políticas, produtos e serviços específicos voltados à colaboração acadêmica. O projeto da UFMG toma como princípio norteador a perspectiva da cidadania científica que de acordo com Moura (2012),

refere-se à possibilidade de, no âmbito dos direitos evocados no desenvolvimento da
cidadania substantiva e no direito difuso, incorporar o direito à educação científica, o
acesso à informação e às controvérsias produzidas pelos atores sociais no âmbito dos
processos criativos científicos e de seus desdobramentos éticos, políticos e mercantis, como
dimensões fundamentais aos processos de democratização da ciência. (MOURA, 2012, p.
21).

Nesse contexto, a Coordenadoria de Políticas de Inclusão Informacional da UFMG (CPINFO) criou e mantém desde o ano de 2010 o repositório Digital UFMG, um serviço de informação que tem por objetivo apoiar o compartilhamento e a disseminação de informações e conhecimentos de interesse público. Dentre os seus objetivos está o de ampliar a visibilidade da produção científica institucional, a circulação dos saberes e o acesso integrado aos documentos eletrônicos gerados no processo da pesquisa científica, ensino e extensão.

O serviço integra o Colaboratório Digital da UFMG, um espaço colaborativo online desenvolvido com o propósito de estimular a cooperação acadêmica e o acesso aberto ao conhecimento produzido no âmbito da Universidade. O projeto tem ainda por objetivo estimular o desenvolvimento e a experimentação de novas abordagens e produções colaborativas, modelos inovadores de comunicação e de divulgação científica e o compartilhamento de ferramentas, dispositivos e metodologias de ensino, pesquisa e extensão na interface Universidade-Sociedade.

Desde o início, o Repositório Digital da UFMG adotou como estratégia a sensibilização e a integração dos profisssionais da informação da Universidade, pois a colaboração desse segmento é fundamental para o sucesso da iniciativa. Nesse sentido, $\mathrm{O}$ Colaboratório Digital UFMG conta hoje com a parceria do Sistema de Bibliotecas e agregará em breve, dentre os produtos e serviços ofertados, a Biblioteca Digital de Teses e Dissertações. Durante o período de implantação foram realizadas inúmeras atividades que tiveram por objetivo compor a política de funcionamento do repositório, assim como o ambiente digital e suas principais funcionalidades. Na configuração de seus produtos e serviços o Repositório tem como princípio a adoção de tecnologia de acesso aberto.

A equipe responsável pelo projeto tem também buscado parcerias nacionais e internacionais que levem ao fortalecimento do acesso aberto à informação em nível nacional. Nesse sentido, participou da $2^{\text {a }}$ Conferência Luso-Brasileira de Acesso Aberto, realizada no 
Rio de Janeiro em 2011 e com a presença das diversas iniciativas desenvolvidas no âmbito dos países de língua portuguesa.

No período de 2012-2013 a equipe tem como meta promover o povoamento do espaço digital criado e a ampliação das coleções, através de cursos de formação e sensibilização da comunidade acadêmica para a política de auto-arquivamento dos documentos eletrônicos gerados nas atividades conexas de ensino, pesquisa e extensão com base em princípios éticos de gestão e difusão da informação.

\subsection{Repositório Interinstitucional "Saber" (RIS) de Moçambique}

A iniciativa da criação de RIS de acesso livre à informação científica em Moçambique, lançado em Novembro de 2010, surgiu a partir de um esforço ${ }^{7}$ conjunto de três instituições de ensino superior e de pesquisa (IESI), nomeadamente, a Universidade Eduardo Mondlane (www.uem.mz), a Universidade Politécnica "A Politécnica" (www.apolitecnica.ac.mz) e o Centro de Formação Jurídica e Judiciária (www.cfjj.org.mz), que se tornou referência em projetos voltados ao movimento do acesso livre ao conhecimento no país. Portanto, a implementação do RIS é uma das formas que foi encontrada para que as instituições acadêmicas e de pesquisa para a disseminação e divulgação das suas publicações científicas, com vista a minimizar a falta de visibilidade de sua produção intelectual.

A história da produção científica nas IESI é relativamente recente em Moçambique. A pesquisa (ou produção) científica em Moçambique é ainda "nascente", os institutos de pesquisa são recentes e os pesquisadores são geralmente jovens recém formados. $O$ financiamento da pesquisa resulta de apoios externos. Apesar disso, nos últimos dez anos, nota-se que há uma grande preocupação, por parte do governo, em desenvolver áreas como inovação do ensino, pesquisa e desenvolvimento (P\&D). Por um lado, o governo procura incentivar a produção científica e tecnológica como meio de formação de solução aos problemas com relevância para o desenvolvimento do país.

Embora limitados, os resultados e o impacto da pesquisa são já significativos, mas a sua difusão ainda é bastante restrita. Foi, a partir dessa preocupação, que se criou o Repositório Interinstitucional "Saber".

A criação do RIS teve como principais objetivos; recolher, agregar e indexar conteúdos acadêmicos e científicos existentes nas instituições de ensino superior e de

\footnotetext{
${ }^{7}$ Com o apoio financeiro da SIDA/SAREC (Agência Sueca para o Desenvolvimento Internacional) e do Projeto QIF do Banco Mundial e o apoio técnico da Universidade de Minho (Portugal). 204

Enc. Bibli: R. Eletr. Bib. Ci. Inf., ISSN 1518-2924, Florianópolis, v. 17, n. esp. 2 - III SBCC, p.195-210, 2012.
} 
pesquisa em Moçambique que possam ser disponibilizados em acesso livre; e disponibilizar a pesquisa, descoberta, localização e acesso a documentos de caráter científico e acadêmicos produzidos em Moçambique e/ou sobre Moçambique. Assim, o RIS é o primeiro repositório institucional de caráter interinstitucional, criado com o propósito, de atender, não só as instituições de ensino superior, como também aos institutos ou centros de pesquisa. O RIS foi implantando com a finalidade de criar um ambiente digital no qual a produção científica de docente, pesquisador e discente, oriunda de diferentes comunidades acadêmicas e científicas, possa ser publicada, conhecida e utilizada por outros membros da comunidade e pela sociedade moçambicana em geral.

Desta forma, no RIS, no início da sua construção, previa o depósito de seguintes materiais, em todas as comunidades: manuscritos, artigos de periódicos (pre-prints e postprints), materiais de ensino (manuais didáticos, textos sobre determinadas obras, etc.), monografias, partes de livros, relatórios técnicos, dissertações, teses e vídeos. Passados aproximadamente três anos, desde o lançamento, podemos encontrar materiais de ensino, monografias, dissertações e teses.

Na configuração do RIS adotou-se a plataforma $D_{s p a c e}{ }^{8}$, uma das mais conhecidas para criação de repositórios institucionais no meio científico. Essa plataforma possibilita: (a) recolher e descrever documentos digitais, de acordo com um workflow adaptável aos processos específicos de uma comunidade; (b) distribuir os documentos digitais da instituição na Web, possibilitando a pesquisa e obtenção da informação; (c) preservar os documentos digitais em longo prazo. (CARVALHO et al., 2006).

Desta forma, estruturalmente, o RIS está organizado em comunidades, subcomunidades e coleções. Cada comunidade representa uma das instituições que tenha conteúdo indexado no repositório. As sub-comunidades correspondem às faculdades, escolas, departamentos ou outros setores das instituições que disponibilizam informação científica neste repositório. As coleções são as espécies documentais a serem disponibilizadas pelas diferentes comunidades e sub-comunidades. Relativamente, ao depósito de textos e documentos é feito mediante solicitação, qualquer instituição de ensino superior ou de

\footnotetext{
${ }^{8}$ O Dspace é o resultado de um esforço conjunto do Massachusetts Institute of Technology (MIT) e da HewlettPackard (HP), desenvolvido com a finalidade de coletar, preservar e disseminar documentos digitais produzidos por docentes e pesquisadores do MIT. Posteriormente, adaptado por um conjunto de oito universidades americanas, o Dspace é hoje disponibilizado sob a forma de um produto open source, que pode ser livremente adaptado e expandido funcionalmente, nos termos da BSD (Berkeley Standard Distribution License Open Source License). Escrito em Java, roda em qualquer tipo de sistema operacional do tipo Unix, servidor web Apache e base de dados PostgressSQL. Utiliza metodologia de arquivos abertos (Open Access Initiative) e padrões internacionais, como o Dublin Core.
} 
pesquisa, em Moçambique, pode submeter os seus conteúdos. A inclusão de conteúdos é feita mediante solicitação acompanhada por termo de autorização com a assinatura do autor. A qualidade dos materiais depositados é da responsabilidade das comunidades que o publicam.

Assim, o RIS visa contribuir para uma nova era na comunicação científica no país. A metodologia de funcionamento do Repositório "Saber" adotada possibilita um maior intercâmbio e engajamento das instituições na coleta, gestão, divulgação e preservação da sua própria produção literária. $\mathrm{O}$ conhecimento gerado por estes órgãos tem sido utilizado não só para gerar novos conhecimentos, como também para mostrar o seu potencial competitivo nas áreas onde atua. No entanto, o compartilhamento desse conhecimento com a sociedade em geral é um dos ganhos mais visíveis e importantes deste tipo de serviço em Moçambique, nos últimos anos.

\section{CONSIDERAÇÕES FINAIS}

O acesso livre à informação científica no Brasil e em Moçambique, via repositórios institucionais, é, sem dúvida, uma alternativa viável para desenvolvimento da pesquisa, ensino e extensão. As instituições de ensino superior e de pesquisa precisam adotar políticas de livre acesso à informação científica e estratégias que contribuam para a melhoria do acesso e a difusão dos resultados da produção científica. Um esforço é exigido aos dirigentes das universidades e do setor privado, bibliotecários, pesquisadores, agências de financiamento e de governo dos países para implementar e explorar plenamente o acesso livre à informação científica.

No Brasil e em Moçambique, o acesso livre aos Portais de periódicos das CAPES e outros portais científicos de acesso aberto, são exemplos bem sucedidos de disponibilização de informação técnico-científica publicada em meios eletrônicos sem custo para os alunos, docentes e pesquisadores. Neste contexto, apesar de esses recursos estarem disponíveis e estudos de sua utilização e aceitação serem realizados, ainda há certo desconhecimento entre as comunidades acadêmicas sobre a disponibilidade e os princípios gerais destas ferramentas. Acredita-se que a ampliação das estratégias de comunicação e expansão das fontes eletrônicas de acesso aberto podem contribuir na redução das assimetrias verificadas na produção e difusão do conhecimento.

Ressalta-se ainda que os autores são unânimes ao afirmaram que "os repositórios institucionais apresentam novas estratégias para o compartilhamento da informação científica 206 
e são importantes recursos para a visibilidade dessa informação. A sua implantação deve ser realizada com base em diretrizes estruturadas a partir de estudos dos aspectos técnicos, do ambiente e da comunidade envolvida".

Dessa forma, os repositórios institucionais, além de "expandir o acesso à pesquisa, reafirmar o controle sobre o saber pela academia, reduzir o monopólio dos periódicos científicos", entre outras mudanças significativas no sistema de comunicação científica, têm o potencial de "servir como indicadores tangíveis da qualidade de uma instituição e de demonstrar a relevância científica, social e econômica de suas atividades de pesquisa, aumentando a visibilidade, o status e o valor público da instituição”. (CROW, 2002). Em outras palavras, como ressalta Lawrence (2003), "repositórios institucionais são uma manifestação visível da importância emergente da gestão do conhecimento na educação superior". Lawrence prevê que, em longo prazo, é provável que "o impacto dos repositórios institucionais mude muitas das suposições a respeito de como a produção intelectual é gerida por indivíduos, seus colegas e a academia, além de como a própria pesquisa é conduzida".

Do ponto de vista das experiências analisadas nesse trabalho, parece ser evidente que implantação dos repositórios institucionais pode contribuir efetivamente na horizontalização das relações entre os pesquisadores das diversas áreas do conhecimento e na implementação de novas formas de acesso à produção científica. Além disso, estas iniciativas podem levar a maior visibilidade das pesquisas realizadas em países em desenvolvimento com repercussões positivas nas ações de todos os atores sociais envolvidos. 


\section{REFERÊNCIAS}

BAPTISTA, Ana Alice. Comunicação científica: o papel da Open Archives Initiative no contexto do acesso livre. Enc. Bibli: R. Eletr. Bibliotecon. Ci. Inf., Florianópolis, n. esp., 2007.

BERLIM Declaration, 2003. Disponível em: http://oa.mpg.de/openaccessberlin/berlindeclaration.html. Acesso em 12 Nov. 2009.

BIANCO, S. et al. Towards Open Access publishing in high energy physics: report of the SCOAP working party. Geneva: CERN, 2007.

BUDAPEST Open Access Initiative, 2002. Disponível em: http://www.soros.org/openaccess. Acesso em 12 nov 2009.

CANESSA, Enrique; ZENNARO. Science dissemination using Open Access: a compendium of selected literature on Open Access. Trieste, ICTP, 2008.

CARDOSO, Gustavo et al. As políticas de Open Access: res publica científica ou autogestão? Sociologia, Problemas e Práticas, n. 60, p. 53-67, 2009.

CARVALHO, Maria Carmen Romcy de et al. O repositório institucional como recurso de apoio à gestão do conhecimento da Universidade Católica de Brasília. In: CONFERÊNCIA IBEROAMERICANA DE PUBLICAÇÕES ELETRÔNICAS NO CONTEXTO DA COMUNICAÇÃO CIENTÍfICA, 1, 2006, Brasília. Anais... Brasília: Universidade de Brasília, 2006.

COSTA, S. Filosofia aberta, modelos de negócios e agências de fomento: elementos essenciais a uma discussão sobre o acesso aberto à informação científica. Ciência da Informação, v. 35, n. 2, p. 39-50, maio/ago., 2006. Disponível em: http://www.ibict.br/cionline/viewarticle.php?id=926. Acessado em: 23 fev. 2007.

COSTA, Sely Maria de Souza; LEITE, Fernando César Lima. Repositórios institucionais: potencial para maximizar o acesso e o impacto da pesquisa em universidade. In: CONFERÊNCIA IBEROAMERICANA DE PUBLICAÇÕES ELETRÔNICAS NO CONTEXTO DA COMUNICAÇÃO CIENTÍFICA, 1, 2006, Brasília. Anais... Brasília: Universidade de Brasília, 2006.

CRESPO, Isabel Merlo; CORRÊA, Cynthia H. W. Acesso livre à comunicação científica: a experiência do Scielo. Revista F@ro: Estudios, n. 6, p. 1-6, 2006. Disponível em: http://web.upla.cl/revistafaro.

CROW, R. The case for institutional repositories: a SPARC position paper. [S.1.]: The Scholarly Publishing and Academic Resources Coalition, 2002. 37 p. Disponível em: http://www.arl.org/sparc/IR/ir.html. Acessado em: 10 mar. 2006.

GENONI, Paul. Content in institutional repositories: a collection management issue. Library Management, v. 25, n. 6/7, p. 300-306, 2004. 
GIBBONS, Susan. Establishing an institutional repository. Chicago: ALA, 2004. 68p. Disponível em: http://1624.brianwinterman.com/gibbons.pdf. Acessado em: 18 jul. 2007.

HOCKX-YU, H. Digital preservation in the context of institutional repositories. 2006. Disponível em: http://eprints.rclis.org/archive/00007351/01/DPinIRs_Final.pdf. Acessado em: 19 fev. 2007.

KURAMOTO, Hélio. Acesso livre à informação científica: novos desafios. Liinc em Revista, Rio de Janeiro, v. 4, n. 2, p. 154-157, set. 2008. Disponível em: http://www.ibict.br/liinc/

KURAMOTO, Hélio. Os open archive e as políticas públicas para a informação científica. In: SIMPÓSIO INTERNACIONAL DE BIBLIOTECAS DIGITAIS, 3, 2005, São Paulo. Disponível em: http://bibliotecascruesp.usp.br/bibliotecas/APRESENT/Helio Kuramoto.ppt.

LAWRENCE, Steve. Free online availability substantially increases a paper's impact. Nature webdebates. Disponível em: http:/www.nature.com/nature/debates/eaccess/Articles/lawrence.html. Acessado em: 20 nov. 2004.

LEITE, Fernando César Lima. Como gerenciar e ampliar a visibilidade da informação científica brasileira: repositórios institucionais de acesso aberto. Brasília, DF: IBICT, 2009.

LEITE, Fernando César Lima; COSTA, Sely. Repositórios institucionais como ferramentas de gestão do conhecimento científico no ambiente acadêmico. Perspect. Ciênc. Inf., Belo Horizonte, v. 11, n. 2, p. 206-219, mai./ago. 2006.

LYNCH, C. A. Institutional repositories: essential infrastructure for scholarship in the digital age. ARL Bimonthly Report, n. 226, feb. 2003. Disponível em: http://www.arl.org/newsltr/226/ir.html. Acessado em: 10 mar. 2006.

MANGUE, M. V. Consolidação do processo de informatização em sistemas de bibliotecas de universidades da África do Sul, Brasil e Moçambique. 2007. 289f. Tese (Doutorado em Ciência da Informação - Programa de Pós-Graduação em Ciência da Informação, Universidade Federal de Minas Gerais, 2007).

MARON, Nancy L. SMITH, K. Kirby. Current models of digital scholarly communication. Available in: http://www.arl.org/bm doc/current-models-report.pdf.

MORENO, Fernanda Passini; LEITE, Fernando César Lima; ARELLANO, Miguel Ángel Márdero. Acesso livre a publicações e repositórios digitais em ciência da informação no Brasil. Perspect. Ciênc. Inf., Belo Horizonte, v. 11, n. 1, p. 82-94, jan./abr. 2006.

MOURA, Maria Aparecida. Informação e conhecimento em redes virtuais de cooperação científica: necessidades, ferramentas e usos. Datagramazero, v. 10, n. 2, abr. 2009.

MUELLER, Suzana Pinheiro Machado. A comunicação científica e o movimento de acesso livre ao conhecimento. Ciência da Informação. Brasília, v. 35, n. 2, p.27-38, maio/ago. 2006. 
ONDARI-OKEMWA, Ezra. Scholarly publishing in Sub-Saharan Africa in the twenty century: challenges and oportunities. First Monday, v. 12, n. 10, oct. 2007.

PAPPALARDO, Kylie. Understanding open access in the academic environment: a guide for authors. Disponível em: http://www.oaklaw.qut.edu.au/materials/licence injector/Author_Guide_lic.pdf.

REPOSITÓRIO Científico de Acesso Aberto de Portugal - RCAAP. Disponível em: http://projecto.rcaap.pt/. Acessado em: 31 mar. 2012.

REPOSITÓRIO Digital da UFMG. Disponível em: http://www.ufmg.br/repositoriodigital

RODRIGUES, E. et al. RepositóriUM: criação e desenvolvimento do Repositório Institucional da Universidade do Minho. Disponível em: https://repositorium.sdum.uminho.pt/bitstream/1822/422/1/BAD artigo+-+Final.pdf.

RUMSEY, S. The purpose of institutional repositories in UK higher education: a repository manager's view. International Journal of Information Management, v. 26, n. 3, p. 181186, 2006. Disponível em: http://eprints.lse.ac.uk/archive/00000800.

SANTOS, J.; TEIXEIRA, C.; PINTO, J. S. eABC: um repositório institucional virtual. Disponível em: http://vecpar.fe.up.pt/xata2005/papersfinal/31.pdf. Acessado em: 11 mar. 2006.

SANZ VALERO, Javier; VEIGA DE CABO; Jorge; DAVID CASTIEL, Luis. A iniciativa Open Access no acesso à informação técnico-científica nas ciências da saúde. RECIIS - R. Eletr. de Com. Inf. Inov. Saúde. Rio de Janeiro, v. 1, n. 1, p. 19-26, jan./jun. 2007.

SAYÃO, Luis et al. (Org.). Implantação e gestão de repositórios institucionais: políticas, memória, livre acesso e preservação; Salvador: EDUFBA, 2009.

TOMAÉL, Maria Inês; SILVA, Terezinha Elisabeth da. Repositórios institucionais: diretrizes para políticas de informação. In: ENANCIB - ENCONTRO NACIONAL DE PESQUISA EM CIÊNCIA DA INFORMAÇÃO, 8, 2007. Anais... Salvador: ENANCIB, 2007.

VEIGA DE CABO, Jorge; SANZ-VALERO. A iniciativa Open Access no acesso à informação técnico-científica nas Ciências da Saúde. R. Eletr. de Com. Inf. Inov. Saúde, v. 1, n.1, p. 19-26, jan./jun., 2007.

WEITZEL, Simone da Rocha. O papel dos repositórios institucionais e temáticos na estrutura da produção científica. Em Questão, Porto Alegre, v. 12, n. 1, p.51-71, jan./jun. 2006.

WREN, Jonathan D. Open access and openly accessible: a study of scientific publications shared via the internet. Information e practice, april, 2005. 